\title{
Editorial
}

\section{Dose Myocardial MIBI Washout Rate Has Relation to Risk Stratification of Coronary Artery Disease.}

\section{Moustafa, $M$ and Omar, $M$.}

Kasr Al-Ainy Center of Oncology and Nuclear Medicine, Faculty of Medicine,

Cairo University, Egypt.

The risk assessment algorisms of CAD differs in differs according to the parameters they use ${ }^{(1)}$. Some use, in addition to the major risk factors, additional markers such as C-reactive protein (CRP) or family history of early vascular disease ${ }^{(2)}$. However, still all the risk scores have some limitations. Major risk factors were first identified by the Framingham Heart Study (FHS) resulting in better prevention and treatment. The FHS identified both the modifiable and non-modifiable risk factors for CAD. Number of risk scores for men and women are now available to estimate the likelihood of development of $\mathrm{CAD}^{(3)}$.

\section{Non-Modifiable Factors:}

The major non-modifiable factors include age, gender, race and family history. Age is the main risk factor for CAD. The incidence of coronary artery disease rises rapidly with age in men and also in women following the menopause. They may present a difficult challenge in knowing how far to investigate, and there may be practical difficulties with undertaking simple non-invasive tests such as treadmill or cycle exercise. The elderly are also more likely to have silent ischemia and to suffer silent myocardial infarcts ${ }^{(4)}$.

Clinical and population-based studies have demonstrated that genetic factors play important roles in CAD and MI. Dai et al demonstrated that a history of early onset ischemic heart disease (IHD) of first degree relatives was significantly associated with and predicted early onset IHD $\left(<55\right.$ in men and $<65$ in women ${ }^{(5)}$. The subsequent FHS confirmed that a family history of premature $\mathrm{CAD}$, defined as the presence of a first degree relative with a diagnosis of CAD at $<55$ years of age in men and $<65$ years of age in women, is an independent risk factor for CAD. 
The strength of hereditary in determining the risk of CAD increases with an increasing number of affected first-degree relatives and with a younger age of onset (6).

\section{Modifiable Factors}

Covariates related to both obesity and coronary heart disease risk, including hypertension; dyslipidemia and impaired glucose tolerance or non-insulin-dependent diabetes mellitus with insulin resistance and accompanying hyperinsulinemia ${ }^{(7)}$. Long-term longitudinal studies indicate that obesity as such not only relates to but independently predicts coronary atherosclerosis ${ }^{(8)}$.

Type II diabetes mellitus is of increasing prevalence in non-industrialized countries, and a major association of coronary artery disease and of a poor prognosis. These patients are more likely to have diffuse coronary artery disease, and more likely to have silent myocardial infarction and episodes of silent ischemia.

United Type 2 diabetes risk factor even when adjusting for advancing age, hypertension, smoking, hypercholesterolemia, and left ventricular hypertrophy ${ }^{(9)}$. IHD develops at a younger age in diabetics and is more severe and diffuse in age-matched controls.
Premature IHD is noted in insulindependent diabetics.

In NIDDM, risk of developing IHD is $2-4$ times higher than in the general population.

High blood cholesterol occurs when diet is rich in saturated fats or may be inherited (as in Familial Hypercholesterolemia (FH) and Familial Combined Hyperlipidaemia $(\mathrm{FCH}))$. With elevated serum low density lipoprotein (LDL) concentrations, which is the richest source of cholesterol which will deposit in the walls of arteries, that become damaged and may become blocked and subsequently heart attack could result. Although rising blood triglyceride levels are a weak risk factor, it is often coupled with having too little high density lipoprotein (HDL). This combination is commonly associated with premature coronary heart disease. It may be inherited and also occurs in obese individuals. Low levels of HDL in the blood appear to be an important predictor for heart disease ${ }^{(\mathbf{1 0}, \mathbf{1 1})}$. Hypertension induces endothelial dysfunction, exacerbates the atherosclerotic process and it contributes to make the atherosclerotic plaque more unstable. Left ventricular hypertrophy (which is the usual complication of hypertension) increases myocardial oxygen demand, both mechanisms contributing to myocardial ischemia. 
Hypertension is frequently associated to metabolic disorders, such as Insulin resistance with hyperinsulinemia and dyslipidemia, which are additional risk factors of atherosclerosis. Coronary reserve is impaired in patients with essential arterial hypertension in the absence of CHD, which is explained in part by the presence of left ventricular hypertrophy.

It is important to emphasize that complications and mortality of patients suffering a myocardial infarction are greater in hypertensive patients. Treatment should be aimed to achieve optimal values of blood pressure, and all the strategies to treat coronary heart disease should be considered on an individual basis $(\mathbf{1 2}, \mathbf{1 3})$.

Smoking is a risk factor of IHD which is dose-related. Those patients smoking 20 or more cigarettes per day have a 2-3 times greater risk of developing a major coronary event than the general population. Stress can exacerbate symptoms in people with pre-existing heart disease, and can contribute to high blood pressure. Psychological stress is added as an independent risk factor after a majority of research into the field discovered that Type A personality were twice as likely to exhibit CAD as any other personality type ${ }^{(\mathbf{1 4}) .}$
The need to improve diagnosis and risk prediction has prompted the search for novel markers in cardiovascular medicine this allows a more precise planning of potential treatment options.

\section{Diagnosis of CAD:}

ECG is the most accessible and widely used diagnostic tool for patients with coronary heart disease. It is simple, noninvasive, with no any radiation hazards and often very valuable. However, this must be viewed in the light of the history \& physical findings. The main drawback of resting ECG that it could be entirely normal in the presence of severe coronary artery disease and even if it demonstrates the presence of ischemia, ECG cannot confirm the exact anatomical distribution or the severity of damage in the coronary artery involved $^{(\mathbf{1 5}) \text {. }}$

ECG has also a role in risk stratification, revealing signs of previous MI, bundle branch block, left ventricular hypertrophy, conduction defects, and arrhythmias. Stable angina patients with resting ECG abnormalities such as presence of Q waves, persistent ST-T changes, left ventricular hypertrophy, left bundle branch block, second- or third-degree heart block, or atrial fibrillation have worse outcome 
Compared to angina patients with a normal resting ECG. ECG correctly detected significant stenosis with an overall sensitivity $51.5 \%$ and specificity $66.1 \%$. Based on artery analysis, ECG sensitivity is highest for LAD ischemia (37.3\%) and lowest for RCA $(25.8 \%)^{(15)}$.

With introduction of exercise ECG, false negative results have significantly decreased. Exercise ECG test records continuously the ECG during a fixed set of exercises on a motorized treadmill. The exercise lasts 6 to 12 minutes depending on the fitness of the person. Patients with severe anginal attacks, dangerous irregular heart rhythm, heart failure, uncontrolled high blood pressure, should not take the exercise stress test. ECG correctly detected significant stenosis with an overall sensitivity $51.5 \%$ and specificity $66.1 \%$.

Based on artery analysis, ECG sensitivity is highest for LAD ischemia (37.3\%) and lowest for RCA (25.8\%).

Numerous markers of prognosis of the exercise ECG have been identified including exercise capacity, maximum workload achieved, heart rate, and blood pressure response to exercise, as well as evidence of exercise-induced ischemia.
These factors can be incorporated into a risk score such as Duke treadmill score, a well-validated tool to predict annual mortality This score incorporates the exercise time using the standard Bruce protocol in minutes, extent of ST-segment deviation either during exercise or in recovery and the clinical symptoms.

Duke Treadmill Score $=$ Exercise time (minutes) $(5 \times$ ST deviation in $\mathrm{mm})-$ ( $4 \times$ angina index $)$

The angina index $=0$ point for no angina, one point for non-limiting angina, two points for angina that limits exercise.

Duke treadmill score $\geq 5$ predicts low cardiovascular events (99\% 4 year survival, annual mortality $0.25 \%$ ). A score between 4 and -10 suggests intermediate risk and such a group may require further risk stratification with other non-invasive stress test such as myocardial perfusion scan. A score of $<\_10$ indicates high cardiovascular event rates $(79 \% 4$ years survival, 5\% annual mortality) suggesting further investigation with coronary angiography with a view to revascularization is considered ${ }^{(\mathbf{1 6})}$.

Echocardiography: Echocardiography is a noninvasive procedure with total absence of radiation; it is one of the most widely used diagnostic tests in cardiology. 
It describes the anatomy of the heart including chambers size \& wall thickness as well as wall motion changes, Doppler echocardiography assesses the cardiac hemodynamic such as volumes, severity of valvular regurgitation and gradients across the stenotic valves ${ }^{(\mathbf{1 7})}$.

In patients with known or suspected CAD, Two-dimensional echocardiography has become a pivotal first line investigation for the differential diagnosis of acute chest pain syndromes, assessing global and regional left ventricular function as well as evaluating complications of myocardial infarction. Because both myocardial ischemia and infarction produce regional wall motion abnormalities, it is difficult to differentiate between them in patients presenting with acute chest pain. However, it may be helpful in excluding ischemia in patients presenting with chest pain and equivocal ECG. Assessment of regional wall function allows localization of the infarct-related artery. Pathologic studies have demonstrated that if $20 \%$ of the myocardial segment undergoes infarction, there is a reduction in its motion during systole of $>50 \%^{(17)}$.

\section{Stress echocardiography (SE) is} frequently used for the detection of CAD.
However, it usually requires a 40\%-60\% narrowing of the coronary vessel to obtain a measurable reduction in maximal flow which results in significant motion abnormalities. Stress echocardiography not only detects the presence of ischemia but also can assess its magnitude, i.e. the severity of ischemia can be ascertained by the degree of motion impairment from minimal hypokinesia to severe akinesia, up to paradoxical systolic expansion dyskinesia $^{(\mathbf{1 8})}$.

The sensitivity and specificity of SE in the diagnosis of CAD varies with the technique used. The sensitivity is $80 \%, 85 \%$ and $78 \%$ and specificity is $86 \%, 76 \%$ and $91 \%$ for dobutamine, exercise and dipyridamole, respectively. Pooled meta-analyses show that SPECT is more sensitive and SE is more specific in the diagnosis of significant CAD and in the assessment of viability ${ }^{(17)}$. Left ventricular systolic function is accepted as the strongest predictor of longterm survival in subjects with CAD. Historical registry data demonstrates that individuals with coronary disease and LV ejection fraction $<50 \%$ have a significantly worse prognosis than those with preserved LV function, with a clear relationship between degree of LV systolic dysfunction and extent of risk. 
Thus in patients with stable CAD, the finding of left ventricular systolic dysfunction should considerably lower the threshold for further investigation. All patients with stable coronary disease should undergo a baseline clinical assessment, with an echocardiogram to quantify LV function ${ }^{(19)}$.

\section{Myocardial Perfusion Imaging:}

One of the most important advantages of MPS over other techniques is its high prognostic value. A normal MPS study not only suggests the absence of flow-limiting coronary disease, but it is also associated with a low likelihood of non-fatal MI or cardiac death (<1\%/year). Conversely, an abnormal scan indicates the presence of significant $\mathrm{CAD}$, and provides valuable incremental prognostic information that is based on the extent and severity of a perfusion abnormality, as well as the presence of other adverse prognostic signs. Thus the risk stratification and prognosis have become its primary role ${ }^{(\mathbf{2 0 )}}$.

${ }^{99 m}$ Tc MIBI is the first technetium-labeled cardiac perfusion agent to be approved for clinical myocardial perfusion imaging in 1990. It is retained intra-cellular in the region of mitochondria because of its negative trans-membrane potential.
It is extracted by the myocardium with a first-pass efficiency of $60 \%$ and with lengthy myocardial retention (myocardial clearance half-time of $>6$ hours), primarily through binding to cytoplasmic mitochondria.

Advantages to this fixed distribution include the convenience of delaying imaging when necessary without loss of sensitivity. However, lack of significant redistribution also means that imaging of myocardial perfusion under stress and rest conditions requires two separate injections of radiopharmaceutical ${ }^{(21)}$.

Several protocols for cardiac imaging are used for assessment of CAD through detection changes in perfusion in different states of heart. Those include 99mTc twoday protocol or one-day protocol, one-day dual tracer protocol using ${ }^{201} \mathrm{Tl}$ rest ${ }^{99 \mathrm{~m}} \mathrm{Tc}$ agent Stress imaging (22).

Initial assessment of myocardial perfusion includes the presence or absence of perfusion defects in both stress and rest studies for scan interpretation.

After initial assessment of the presence or absence of perfusion defects, a complete evaluation of the stress study includes assessment of the location; size, severity, and likely vascular distributions of the 
Visualized abnormalities, in addition to computer quantitative methods are routinely used in conjunction with image analysis. Perfusion defects caused by CAD are more commonly distal, rather than at the base of the heart.

A true perfusion defect should be seen on more than one cross-sectional slice and in other cross-section planes. Certainty increases with lesion size and the degree or severity of photon deficiency related to degree of coronary stenosis ${ }^{(\mathbf{2 3})}$.

Relative perfusion is often presented in a 2D polar map or bull's-eye display generated with circumferential slice count profiles obtained from the short-axis SPECT slices, with the apex at the center of the display and the base of the ventricle at the periphery. Stress-rest difference polar maps are commonly used to analyze for reversible ischemia. Gated SPECT analysis of wall motion and thickening is standard. Calculation of a LVEF is obtained by measuring the change in size of the ventricular cavity during the cardiac cycle using edge detection algorithms ${ }^{(24)}$.

Stunned Myocardium, occur after a transient period of severe ischemia followed by reperfusion, delayed recovery of regional left ventricular function.
The ischemic episode is not severe enough to cause necrosis. This is seen after thrombolysis or angioplasty in patients who have had acute coronary occlusion. Tissue in the affected perfusion watershed is viable and accumulates the radiopharmaceutical immediately after reperfusion. Tracer uptake indicates viability, but the myocardial segment is akinetic. If it is only stunned and not infarcted, wall motion will improve with time. Stress induced transient ischemic dilation and post stress gated SPECT ventricular dysfunction may be manifestations of stunned myocardium ${ }^{(25)}$.

Hibernating Myocardium, although the scintigraphic findings of regional hypoperfusion at stress and rest associated with regional myocardial dysfunction are usually due to myocardial infarction, up to $20 \%$ of patients with these findings do not have infarction, but rather severe chronic ischemia or hibernating myocardium, associated with several hypo-perfusional such status related; the myocytes show preserved cell membrane integrity and sufficient metabolic activity to maintain cellular viability but not contractility, i.e. the myocardium can benefit from coronary revascularization with improvement in cardiac function and reduction in annual mortality ${ }^{(25)}$. 
The reported sensitivity of stress myocardial perfusion imaging using $\mathrm{Tc}$ MIBI ranged from $80 \%$ to $95 \%$ and the specificity from $70 \%$ to $90 \%$. Sensitivity for the diagnosis of CAD is considerably greater for stress myocardial perfusion scintigraphy than the exercise treadmill study alone ( $88 \%$ vs. $75 \%$ ). Specificity is similar $(77 \%)$. The accuracy of vasodilator versus exercise stress is very similar. The normalcy rate is the frequency of normal test results in patients with a low likelihood of CAD, based on Bayesian analysis using age, sex, symptom classification, cholesterol, and results of noninvasive stress testing. Normalcy rates of $90 \%$ or greater have been reported for SPECT myocardial perfusion scintigraphy ${ }^{(20)}$.

Indicators of adverse outcome and prognosis include multiple and large reversible defects, multiple and large irreversible defects, reversible perfusion defects at low level exercise, increased lung-to-heart ratio after stress and transient left ventricular cavity dilatation after exercise.

\section{Multiple Perfusion Defects}

Perfusion defects in more than one coronary artery distribution area indicate multiple vessel disease. Prognosis worsens with increasing number and size of perfusion defects.
Not all significant coronary artery stenosis are always seen on stress perfusion scans. Stress-induced ischemia of the most severe stenotic lesion limits further exercise, and thus other stenosis may not be seen and multiple vessel disease may be underestimated. Three-vessel balanced disease may not be seen at all ${ }^{(24)}$.

\section{Transient Ischemic Dilation}

The normal cardiac response is to dilate during stress and return to normal size promptly with cessation of exercise. Post stress ventricular dilation is abnormal and suggests multi-vessel disease. Other explanation for this finding is myocardial stunning during stress or widespread subendocardial ischemia ${ }^{(24)}$.

\section{Thallium-201 Lung Uptake}

Exercise-induced ${ }^{201} \mathrm{Tl}$ lung uptake is a poor prognostic sign caused by stress induced ventricular dysfunction, elevated left ventricular end-diastolic pressure, and pulmonary capillary wedge pressure. Lungto-myocardial activity ratios $>0.5$ are abnormal. Lung uptake with Tc-99m agents is less reliable because of normal lung activity.

\section{Myocardial MIBI Washout}

It has been considered that MIBI bound to myocardium tends remain for a relatively long period of time without redistribution as in thallium $201^{(\mathbf{2 6})}$. 
However, it was observed in certain circumstances that there is acceleration of myocardial MIBI washout which is called reverse redistribution ${ }^{(27)}$.

Reverse redistribution pattern is defined as either the worsening of an initial perfusion defect or the appearance of a new perfusion defect on the delayed images.

It is considered that the mitochondrial membrane potential and MIBI retention ability are significantly involved in this phenomenon i.e. change in the membrane potential significantly affect MIBI retention in the myocardium ${ }^{(28)}$.

Physiologically, mitochondria are responsible to produce adenosine triphosphate (ATP) to ensure myocardial function and contractility under the normal circumstance of adequate oxidative capacity. The retention of MIBI in myocyte is highly related to normal mitochondrial function to maintain the electrochemical potential on the exterior surface of mitochondria. In ischemic myocardium, because of decrease in oxygen supply, the aerobic metabolism of ATP generation is deactivated and the mitochondrial membrane eventually becomes depolarized and could not any longer preserve the electronegative potential, leading to exhibit fast clearance of MIBI ${ }^{(29)}$.
It is known that MIBI stress SPECT disclosed coronary stenosis by the fact of difference in myocardial perfusion in stress compared to rest status, while MIBI delayed SPECT was believed to reveal the ischemia by detection disorder of mitochondrial function, accumulation of $\mathrm{Ca} 2+$ in the mitochondria of the ischemic myocardial cells leads to significant dilatation of the mitochondria, decreasing mitochondrial activity and ATP-synthesis ability that cause acceleration of MIBI washout. However, myocardial mitochondrial membrane disorder does not necessarily correlate with coronary stenosis, and MIBI washout could be accelerated by coronary artery spasm in reversible ischemia. This suggests that the severity of coronary stenosis and the time course strongly contribute to the occurrence of mitochondrial disorder ${ }^{(30)}$.

The interval of early or delayed image acquisition, the visual evaluation criteria and the calculation method for washout rate (WR) are not standardized in institutions, and the washout evaluation method is still debated. WR using a planar image may be an effective index when the washout is determined in the whole myocardium in patients with myocardial disease. 
However, since the washout of MIBI is increased only in a region that is subject to a coronary artery occlusion in patients with ischemic heart disease, it is difficult to evaluate the washout using global washout (GWR) in the whole myocardium. Therefore, the method for assessing washout of MIBI by visual evaluation using short-axis, horizontal long-axis and vertical long-axis slice images after reconstruction is adopted in patients with ischemic heart disease ${ }^{(31)}$.

In a different method, a polar map for short-axis images is used to prepare a coronary artery dominance map based on the myocardial maximum counts from the apex to the basal area, and a region with decreased tracer accumulation is regarded as an abnormal region in comparison with a normal area with enhanced washout. However, a standard index for washout evaluation in ischemic heart disease has not been established and establishment of such an index will significantly contribute to the diagnosis in nuclear cardiology and reduce variation in the diagnosis among institutions ${ }^{(32)}$.

MIBI reverse redistribution was observed in a high percentage of patients with coronary artery stenosis, and MIBI delayed SPECT was more sensitive to evaluate myocardial damage patients with greater than $75 \%$ but less than $90 \%$ stenosis. Similarly reverse redistribution frequently occurs in salvaged regions in patients subjected to successful revascularization for AMI at an early stage which could attribute to mitochondrial disorder due to ischemia/reperfusion injury ${ }^{(28)}$.

In Takeishi et al, Myocardial SPECT with 99mTc-MIBI was performed in 27 patients with acute myocardial infarction within 1 week after the onset. 23 patients underwent PTCA and 4 patients did not. Out of 22 patients with successful PTCA, WR of 99mTc-MIBI was observed in 15 patients $(68 \%)$. Persistent defects (PD) were seen in twelve patients (seven patients with successful PTCA, one patient with unsuccessful PTCA, and 4 patients who did not receive angioplasty). In patients with WR, regional uptake of $99 \mathrm{mTc}-\mathrm{MIBI}$ in the area of myocardial infarction decreased from $54 \%+/-10 \%$ in the early images to $43 \%+/-8 \%$ in the delayed images $(\mathrm{p}<$ 0.01). Technetium-99m-MIBI clearance from the myocardium was faster in the infarct area than in the normal area $(26 \%$ $+/-7 \%$ versus $9 \%+/-6 \%, p<0.01)$.

Coronary arteriography performed one month later revealed that the patency of the infarct related artery was seen in all 
Patients with WR and 50\% (6/12) in those with PD $(\mathrm{p}<0.01)$.

The extent and severity of a wall motion abnormality were less in patients with WR than in those with PD (extent: $24 \pm 10$ versus $36 \pm 9, \mathrm{p}<0.01$; severity: $-2.7 \pm$ 0.4 versus $-3.4 \pm 0.6, \mathrm{p}<0.01)^{(32)}$.

It has been well established that coronary artery spasm plays an important role in the pathogenesis of angina pectoris and acute coronary syndrome. However, the precise mechanism responsible for coronary spasm still remains to be elucidated. Coronary artery spasm occurs most often in patients at rest, particularly from midnight to early morning, and patients with coronary spastic angina often demonstrate abnormal left ventricular regional wall motion, probably due to myocardial stunning after repetitive coronary spasms. Reverse redistribution of 99mTc-MIBI is evident in patients with coronary artery disease resulting from coronaries stenosis, 99mTc-MIBI redistributed is also reported in myocardial segments with coronary spasm, i.e. enhanced washout of $99 \mathrm{mTc}-\mathrm{MIBI}$ was observed in coronary spastic angina and might suggest that the ability of myocyte to retain the tracer was impaired in viable but damaged myocardium, it is often associated with left ventricular wall motion abnormalities, it may be attributed to the close relation between repetitive brief ischemia and myocardial stunning in patients with coronary spastic angina ${ }^{(33)}$.

A study by Souichi et al was performed on 30 patients diagnosed to have coronary spastic angina30 patients diagnosed as coronary spastic angina and ten control subjects. Out of 30 patients, 17 (57\%) and $24(80 \%)$ patients demonstrated decreased 99mTc-MIBI uptake on early and delayed images, respectively. In these 24 patients, 22 out of 26 segments $(85 \%)$ with decreased 99mTc-MIBI uptake were regions with coronary spasm induced by intra-coronary ergonovine injection.

Reverse redistribution of $99 \mathrm{mTcMIBI}$ was observed in 17 of 30 patients $(57 \%)$ with coronary spastic angina. Regional washout rate of $99 \mathrm{mTc}$ MIBI was calculated and compared among normal control subjects, normal and spastic segments of patients with coronary spastic angina. Washout rate of $99 \mathrm{mTc}-\mathrm{MIBI}$ from spastic segments was higher than those from non-spastic segments and normal subjects (16 $\pm 2 \%$ vs. $11 \pm 5 \%$ and $10 \pm 3 \%, \mathrm{p}<0.01)$.

This study used quantitative methods and calculated segmental uptake and washout of $99 \mathrm{mTc}-\mathrm{MIBI}$ by placing region of interest (ROI) on each myocardial segment 
of the slice, and then it was analyzed and expressed as a percent of the maximal counts in the left ventricle. ${ }^{(33)}$.

Mostafa et al, study included 50 patients with suspected or diagnosed CAD. Out of the 50 patients undergoing myocardial perfusion SPECT imaging; 22 patients had normal perfusion (SSS <4) and 28 patients had abnormal perfusion (SSS $\geq 4$ ). The abnormal group includes 20patients with reversible induced perfusion defects (SDS >2) and8 patients fixed perfusion defect in both stress and rest studies (SDS < 2).

The analysis of global washout rate was not significant in normal perfusion group as well as patients with irreversible perfusion defects with MIBI washout rate mean value of $7 \pm 5$. While in patient with reversible perfusion defects significant MIBI washout rate with mean value of 22 \pm 3 was reported. The percentage of washout rate in the region of LAD vascular territory showed mean value of $21.18 \pm$ 7.2, for LCx vascular territory showed mean value of $17.02 \pm 2.6$, while in region supplied by RCA vascular territory had mean value of $19.17 \pm 3.86$. Also it showed a significant positive linear correlation between the rate of washout and the percentage of reversibility of stress induced perfusion lesions in three vascular territories (LAD vascular territory $r=0.69$, for $\mathrm{LCx}$ vascular territory $=\mathrm{r}=0.66$ and for LAD vascular territory $=r=0.77$ ). They concluded that patients with reversible perfusion defects had significantly higher rate of washout compared to patients with normal perfusion and patients with irreversible perfusion defects and this higher washout rate is directly correlated to the degree of reversibility ${ }^{(34)}$.

Komita et al study includes seventeen patients in different stages of dilated cardiomyopathy and six healthy control subjects were studied. The myocardial washout rate of $99 \mathrm{mTc}-\mathrm{MIBI}$ was calculated in patients with non-ischemic $\mathrm{CHF}$ and compared with biventricular parameters obtained from first pass and ECG-gated myocardial perfusion SPECT data. The WR was positively correlated with the end diastolic volume (EDV) index $(\mathrm{r} 2=0.216)$, the end-systolic volume $(\mathrm{ESV})$ index $(\mathrm{r} 2=0.234)$, the summed motion score $(\mathrm{SMS})(\mathrm{r} 2=0.544)$, and the summed thickening score (STS) $(\mathrm{r} 2=$ 0.656); it was negatively correlated with the left ventricular ejection fraction (LVEF) $(\mathrm{r} 2=0.679)$. This study concluded that myocardial washout rates in $\mathrm{CHF}$ patients were significantly higher than those in normal controls.

The myocardial washout rate of $99 \mathrm{mTc}$ MIBI was thought to be a novel marker for the diagnosis of myocardial damage. 
It is also demonstrated that the $99 \mathrm{~m} \mathrm{Tc}$ MIBI WR correlated inversely with functional cardiac parameters using myocardial perfusion imaging (MPI) in patients with idiopathic dilated cardiomyopathy $\quad{ }^{(35)} . \quad{ }^{99 m}$ Tc $\quad$ MIBI scintigraphy might be a valuable molecular imaging tool for the diagnosis and evaluation of myocardial damage or dysfunction severity of CAD. The application of Tc MIBI washout rate as an additional function myocardial perfusion parameter may be used of risk assessment in CAD.

\section{REFERENCES:}

1. Cappuccio FP, Oakeshott P, Strazzullo $\boldsymbol{P}$, et al. Application of Framingham risk estimates to ethnic minorities in United Kingdom and implications for primary prevention of heart disease in general practice: cross sectional population based study. Bmj. 325(7375):1271; 2002.

\section{Ridker PM, Paynter NP, Rifai N,} et al. C-reactive protein and parental history improve global cardiovascular risk prediction: the Reynolds Risk Score for men. Circulation. 118(22):2243-51; 2008.

\section{Frohlich J, Al-Sarraf A.} Cardiovascular risk and atherosclerosis prevention. Cardiovascular Pathology. 22(1):16-8; 2013.

\section{Madias JE, Chintalapaly $G$,} Choudry M, et al. Correlates and inhospital outcome of painless presentation of acute myocardial infarction: a prospective study of a consecutive series of patients admitted to the coronary care unit. Journal of investigative medicine: the official publication of the American Federation for Clinical Research.;43(6):56774; 1995.

\section{Dai X, Wiernek S, Evans JP, et al.} Genetics of coronary artery disease and myocardial infarction. World journal of cardiology. 8(1):1; 2016.
6. Kessler T, Erdmann J, Schunkert
$\boldsymbol{H}$. Genetics of coronary artery disease and myocardial infarction-2013. Current cardiology reports. 15(6):368; 2013. 
7. Rimm EB, Stampfer MJ, density subfractions:The Atherosclerosis

Giovannucci $\boldsymbol{E}$, et al. Body size and fat distribution as predictors of coronary heart disease among middle-aged and older US men. American Journal of Epidemiology. 141(12):1117-27; 1995.

\section{Rabkin SW, Mathewson FA, Hsu}

$\boldsymbol{P}-\boldsymbol{H}$. Relation of body weight to development of ischemic heart disease in a cohort of young North American men after a 26 year observation period: the Manitoba Study. American journal of cardiology. 39(3):452-8; 1977.

\section{Haffner SM, Lehto S, Rönnemaa}

T, et al. . Mortality from coronary heart disease in subjects with type 2 diabetes and in nondiabetic subjects with and without prior myocardial infarction. New England journal of medicine. 339(4):229-34; 1998.

\section{Sharrett AR, Ballantyne C, Coady}

$S$, et al. Coronary heart disease prediction from lipoprotein cholesterol levels, triglycerides, lipoprotein (a), apolipoproteins $\mathrm{AI}$ and $\mathrm{B}$, and $\mathrm{HDL}$
Risk in Communities (ARIC) Study. Circulation. 104(10):1108-13; 2001.

11. Assmann $G$, Schulte $H$, Von Eckardstein A, et al.. High-density lipoprotein cholesterol as a predictor of coronary heart disease risk. The PROCAM experience and pathophysiological implications for reverse cholesterol transport. Atherosclerosis. 124:S11-S20; 1996.

12. Chobanian AV, Alexander RW. Exacerbation of atherosclerosis by hypertension. Arch Intern Med. 156:19526; 1996.

\section{Rosendorff C, Lackland DT,} Allison M, et al. Treatment of hypertension in patients with coronary artery disease: a scientific statement from the American Heart Association, American College of Cardiology, and American Society of Hypertension. Journal of the American Society of Hypertension. 9(6):453-98; 2015. 
14. Tresch DD, Aronow WS. Smoking and coronary artery disease. Clinics in geriatric medicine. 12(1):23-32; 1996.

15. Shaw LJ, Peterson ED, Shaw LK, et al. Use of a prognostic treadmill score in identifying diagnostic coronary disease subgroups. Circulation. 98(16):1622-30; 1998.

16. Mark DB, Shaw L, Harrell Jr FE, et al. Prognostic value of a treadmill exercise score in outpatients with suspected coronary artery disease. New England Journal of Medicine. 325(12):849-53; 1991.

17. Shaw LJ, Vasey C, Sawada S, R et al. Impact of gender on risk stratification by exercise and dobutamine stress echocardiography: long-term mortality in 4234 women and 6898 men. European heart journal. 26(5):447-56; 2005.

\section{Bouzas-Mosquera A, Peteiro J,} Álvarez-García N, et al. Prediction of mortality and major cardiac events by exercise echocardiography in patients with normal exercise electrocardiographic testing. Journal of the American College of Cardiology. 53(21):1981-90; 2009.

19. Manyari D. Prognostic implications of echocardiographically determined left ventricular mass in the Framingham Heart Study. The New England journal of medicine. 323(24):1706; 1990.

\section{Cremer $\boldsymbol{P}$, Hachamovitch $\boldsymbol{R}$,} Tamarappoo B, editors. Clinical decision making with myocardial perfusion imaging in patients with known or suspected coronary artery disease. Seminars in nuclear medicine. Elsevier; 2014.

\section{Beller GA, Bergmann SR.} Myocardial perfusion imaging agents: SPECT and PET. Journal of nuclear cardiology. 11(1):71-86; 2004.

\section{Kailasnath $\boldsymbol{P}$, Sinusas AJ.} Comparison of Tl-201 with Tc-99mlabeled myocardial perfusion agents: technical, physiologic, and clinical issues. Journal of Nuclear Cardiology. 8(4):48298; 2001.

\section{Cheitlin MD, Alpert JS, Armstrong} $\boldsymbol{W F}$, et al. ACC/AHA guidelines for the clinical application of echocardiography: a report of the American College of Cardiology/American Heart Association Task Force on Practice Guidelines (Committee on Clinical Application of Echocardiography) developed in collaboration with the American Society of Echocardiography. Circulation. 95(6):1686744; 1997. 
24. Ziessman HA, O'Malley JP, Thrall

JH. Nuclear Medicine: The Requisites EBook: Elsevier Health Sciences; 2013.

\section{Kloner RA, Przyklenk K, Patel B.}

Altered myocardial states: the stunned and hibernating myocardium. The American journal of medicine. 86(1):14-22; 1989.

\section{Okada RD, Glover D, Gaffney T,} et al. Myocardial kinetics of technetium99m-hexakis-2-methoxy-2-methylpropylisonitrile. Circulation. 77(2):491-8; 1988.

\section{Richter W-S, Cordes M, Calder D,}

et $\boldsymbol{a l}$. Washout and redistribution between immediate and two-hour myocardial images using technetium-99m sestamibi. European journal of nuclear medicine. 22(1):49-55; 1995.

\section{Takeishi Y, Sukekawa H, Fujiwara}

S, et al.. Reverse redistribution of technetium-99m-sestamibi following direct PTCA in acute myocardial infarction. The Journal of Nuclear Medicine. 37(8):1289; 1996.

\section{Dedkova EN, Blatter LA.}

Measuring mitochondrial function in intact cardiac myocytes. Journal of molecular and cellular cardiology. 52(1):48-61; 2012.
30. Crane $\boldsymbol{P}$, Laliberté $\boldsymbol{R}$, Heminway

$S$, et al. Effect of mitochondrial viability and metabolism on technetium-99msestamibi myocardial retention. Europeanjournal of nuclear medicine. 20(1):20-5; 1993.

31. Peters A. A unified approach to quantification by kinetic analysis in nuclear medicine. Journal of Nuclear Medicine. 34(4):706-13; 1993.

32. Tanaka R, Simada K. Approach to establishment of a standard index for regional washout of a myocardial perfusion agent. Annals of nuclear medicine. 24(10):713-9; 2010.

33. Yasue H, Omote S, Takizawa A, et al. Coronary arterial spasm in ischemic heart disease and its pathogenesis. A review. Circulation Research.;52(2 Pt 2):I147-52; 1983.

34. Omar M, Moustafa H. Myocardial 99Tc-MIBI washout. Egyptian J Nucl Med. 10(2):1; 2014.

\section{Kumita S-i, Seino Y, Cho K,}

Nakaio H, et al. Assessment of myocardial washout of Tc-99m-sestamibi in patients with chronic heart failure: comparison with normal control. Annals of nuclear medicine. 16(4):237-42; 2002. 